\title{
Impactos psicológicos e físicos em profissionais enfermeiros no enfrentamento da pandemia pela COVID-19
}

\author{
Psychological and physical impacts on professional nurses in coping with the COVID-19 pandemic
}

Impactos psicológicos y físicos en las enfermeras profesionales para hacer frente a la pandemia del

COVID-19

Recebido: 02/05/2021 | Revisado:10/05/2021 | Aceito: 03/07/2021 | Publicado: 15/07/2021

\author{
Izadora Nunes da Silva \\ ORCID: https://orcid.org/0000-0001-6302-6310 \\ Centro Universitário Mario Pontes Jucá, Brasil \\ E-mail: izadoranunes19.in@gmail.com \\ Amauri dos Santos Araujo \\ ORCID: https://orcid.org/0000-0001-7435-5670 \\ Universidade Federal de Alagoas, Brasil \\ E-mail: amauriaraujo.sms@gmail.com
}

\begin{abstract}
Resumo
A pandemia ocasionada pela COVID-19, provocou grandes alterações não apenas na organização dos serviços de saúde, mas no que se refere também às condições de saúde psíquica e física dos profissionais atuantes na linha de frente, em especial dos enfermeiros. Objetivo: Evidenciar as principais implicações físicas e mentais em enfermeiros no enfrentamento da pandemia pela COVID-19. Método: Trata-se de um estudo do tipo revisão integrativa de abordagem qualitativa, utilizando as bases de dados SCIELO, BDENF e LILACS. Resultados e discussões: Os estudos destacam os impactos físicos e psicológicos que interferem no bem-estar dos enfermeiros que atuam em combate à pandemia, entre eles estão a depressão, ansiedade, síndrome de Burnout, tristeza, frustração e entre outros, decorrentes da natureza e condições de trabalho atuais como sobrecarga de trabalho, a escassez de equipamentos de proteção individual, receio de se contaminar, medo da perda dos familiares, de pacientes e colegas do trabalho, isolamento social. Conclusão: No tocante, observa-se os impactos na saúde mental provocados pela pandemia da COVID-19 nos profissionais enfermeiros, trazendo à tona os fatores que levam ao adoecimento psicológico desses profissionais e como eles são experienciados.
\end{abstract}

Palavras-chave: Pandemia; Coronavírus; Enfermagem; Saúde mental.

\begin{abstract}
The pandemic caused by COVID-19 caused major changes not only in the organization of health services, but also in terms of the mental and physical health conditions of professionals working on the front lines, especially nurses. Objective: To highlight the main physical and mental implications for nurses in coping with the pandemic by COVID19. Method: This is an integrative review study with a qualitative approach, using the SCIELO, BDENF and LILACS databases. Results and discussions: The studies highlight the physical and psychological impacts that affect the wellbeing of nurses working to combat the pandemic, including depression, anxiety, Burnout syndrome, sadness, frustration and among others, arising from nature and current working conditions such as work overload, shortage of personal protective equipment, fear of being contaminated, fear of loss of family members, patients and colleagues, social isolation. Conclusion: Regarding the impact on mental health caused by the pandemic of COVID-19 in the nursing professionals, it highlights the factors that lead to the psychological illness of these professionals and how they are experienced.
\end{abstract}

Keywords: Pandemic; Coronavirus; Nursing; Mental health.

\section{Resumen}

La pandemia causada por COVID-19 provocó cambios importantes no solo en la organización de los servicios de salud, sino también en las condiciones de salud mental y física de los profesionales que trabajan en primera línea, especialmente enfermeras. Objetivo: Destacar las principales implicaciones físicas y mentales del enfermero en el afrontamiento de la pandemia por COVID-19. Método: Se trata de un estudio de revisión integradora con enfoque cualitativo, utilizando las bases de datos SCIELO, BDENF y LILACS. Resultados y discusiones: Los estudios destacan los impactos físicos y psicológicos que afectan el bienestar de los enfermeros que trabajan para combatir la pandemia, incluyendo depresión, ansiedad, síndrome de Burnout, tristeza, frustración y entre otros, derivados de la naturaleza y las condiciones laborales actuales como sobrecarga de trabajo, escasez de equipo de protección personal, 
miedo a la contaminación, miedo a la pérdida de familiares, pacientes y compañeros, aislamiento social. Conclusión: Respecto al impacto en la salud mental que ocasionó la pandemia de COVID-19 en los profesionales de enfermería, es posible destacar los factores que conducen a la enfermedad psicológica de estos profesionales y cómo se vivencian.

Palabras clave: Pandemia; Coronavirus; Enfermería; Salud mental.

\section{Introdução}

O advento da nova pandemia ocasionada pela COVID-19, provocou grandes impactos não apenas na organização dos serviços de saúde, mas no que se refere também as condições de saúde psíquica e física dos profissionais que atuam na linha de frente, em especial dos enfermeiros sendo um dos principais profissionais que estão lidando diretamente com a população infectada através da assistência prestada. Sendo assim, este estudo alude acerca dos impactos na saúde física e mental dos enfermeiros no enfrentamento da COVID-19, levando em consideração que não só população geral estão experienciando sentimentos e emoções estressores em virtude da situação vivenciada (Araujo; Comassetto, 2021; Melo et al., 2020; Teixeira, 2020; Saidel, 2020).

Após a descoberta do novo agente do Coronavírus em 31 de dezembro de 2019 devido aos surgimentos dos primeiros casos registrados em Wuhan, na China, que este vírus se tornou uma das grandes preocupações dos sistemas de saúde resultando em nível de alerta em decorrência dos altos índices de propagação e disseminação e primordialmente devido às complicações críticas que pode acarretar. Diante disso, a Organização Mundial da Saúde (OMS) declarou, no mês de janeiro de 2020, que o surto da doença causada pela COVID-19 constituía então uma emergência de saúde pública de importância internacional, requerendo assim um plano de medida urgente para combater essa pandemia (Chate et al., 2020; Teixeira et al., 2020; Schmidt et al., 2020).

Atualmente sabe-se que o atual Coronavírus (SARS-CoV-2) faz parte dos sete coronavírus humanos (HCoVs) já conhecidos, pertencendo a uma família responsável principalmente por quadros respiratórios, sendo relatada como a síndrome respiratória viral mais severa presenciada até hoje. A mesma, pode se manifestar de forma assintomática e/ou sintomática, sendo esta última podendo provocar complicações críticas demandando assim internação hospitalar, e em muitos casos levando até a morte. Dentre os principais sintomas destacam-se a febre, tosse e as dificuldades respiratórias (Araujo; Comassetto, 2021; Seixas, 2021; Teixeira, 2020; Saidel, 2020; Schmidt et al., 2020).

No Brasil, ainda existe há incerteza da ocorrência dos primeiros casos, tendo em vista as características dos surgimentos dos sintomas e a rápida e silenciosa propagação da infecção, entretanto foi confirmada a primeira morte por diagnóstico de COVID-19 em 25 de fevereiro de 2020 em São Paulo (Seixas, 2021). Ademais, desde o início da pandemia já foram registrados 12.573.615 casos confirmados e 313.866 óbitos em todo território nacional segundo os dados fornecidos pela World Health Organization, colocando o Brasil assim entre os países com os maiores índices de diagnósticos de casos no mundo (Seixas, 2021; Schmidt, 2020).

Diante desse cenário, evidencia-se a crítica situação dos profissionais da saúde, em especial dos enfermeiros, observando as intensificações da rotina de trabalho em virtude da pandemia gerando sobrecarga e desgastes físicos, falta de equipamentos de proteção individual, treinamentos e capacitações para lidar com a doença e entre outros fatores que colocam o bem-estar desse grupo em um quadro que merece atenção. Afora as implicações físicas, é preciso considerar também as implicações na saúde mental como focos primários de atenção a esse grupo de modo que não sejam subestimadas ou negligências (Seixas, 2021; Melo, 2020; Schmidt, 2020).

Em linhas gerais, considerando a vulnerabilidade que encontram os profissionais da enfermagem que atuam de direta ou indireta na assistência e combate ao coronavírus ao confronto com seus recursos físicos e psicológicos (Melo, 2020). Surge a necessidade de responder ao seguinte questionamento: Quais são os impactos na saúde física e mental dos enfermeiros no 
enfrentamento da COVID-19? Diante o exposto, este estudo objetiva evidenciar as principais implicações físicas e mentais em enfermeiros no enfrentamento da pandemia pela COVID-19.

\section{Metodologia}

Trata-se de um estudo de abordagem qualitativa do tipo revisão integrativa; pois se entende que a partir desse tipo de pesquisa se obtém uma análise mais detalhada acerca da temática pesquisada, já que possibilita delimitar o assunto e extrair novas informações a partir de dados obtidos dos estudos já publicados. Ademais, a revisão é um recurso que possibilita uma abordagem ampla e sistêmica, fornecendo subsídios para sintetizar conhecimentos, identificar lacunas existentes entre as pesquisas, além de formular conclusões a respeito de uma determinada área de estudo (Araujo et al., 2020; Donato, 2019; Cunha, 2015; Soares et al., 2014; Mendes; Silveira; Galvão, 2008; Whittemore; Knafl, 2005).

A escolha desse tipo de estudo baseou-se na necessidade de reunir e sintetizar os achados referentes ao assunto investigado, com o objetivo de aprofundar e formular novos conhecimentos. Assim, este estudo permeou 06 (seis) etapas preconizadas, sendo estas: formulação da questão norteadora; especificação dos fatores de inclusão e exclusão; extração dos dados nas bases de dados escolhidas; extração dos estudos e aplicabilidade dos fatores de inclusão e exclusão; análise e avaliação dos dados obtidos incluídos para construção da revisão a apresentação/sintetize dos dados produzidos (Araujo et al., 2020; Donato, 2019; Cunha, 2015; Whittemore; Knafl, 2005). A presente revisão teve como questão norteadora: "Quais são os impactos na saúde física e mental dos enfermeiros no enfrentamento da covid-19?”.

As plataformas pesquisadas para obtenção dos dados foram SCIELO (Scientific Electronic Library Online), LILACS (Centro Latino-Americano e do Caribe de Ciências da Saúde) e BDENF (Base de dados de enfermagem), a busca foi realizada entre os meses de março e abril 2021. Como estratégias de investigação, foram utilizados os Descritores em Ciências da Saúde (DeCS): "pandemic", "Coronavirus", "nursing" e "mental health", utilizando o recurso booleano AND, organizando no seguinte agrupamento: "pandemics" and "coronavirus" and "nursing" and "mental health".

Foram incluídos no estudo artigos disponíveis gratuitamente e indexados nas bases previamente estabelecidas, completos e publicados entre fevereiro de 2016 a fevereiro de 2021 no idioma português e inglês, disponíveis na gratuitamente. Já os critérios de exclusão foram cartas ao editor, editoriais, teses e dissertações. Foram considerados apenas uma vez na amostra os artigos encontrados em mais de uma base de dados (duplicados). Com o emprego dos descritores indexados nas bases de dados foram encontrados o total de 38 artigos, após o emprego dos critérios de inclusão e exclusão foram selecionados o total de 22 estudos, sendo que desses após análise 15 foram selecionados para amostra final e após a leitura criteriosa 09 artigos foram aptos para compor esta revisão sistemática, conforme mostra o Quadro 1.

Ao final da busca dos artigos e análise na íntegra, para à melhor compreensão e organização dos dados obtidos nos artigos, foi construindo dois quadros didáticos e expositivos contendo no primeiro as principais informações dos 09 artigos, como: título do artigo e autor; tipo de estudo e ano de publicação, objetivo e principais resultados, e no segundo quadro a base de dados encontrada, o nível de evidência do artigo e o Qualis da revista. Salienta-se que os aspectos éticos relativos às pesquisas científicas foram respeitados. 
Tabela 1 - Cruzamentos dos descritores nas bases de dados selecionadas. Maceió - AL, 2021.

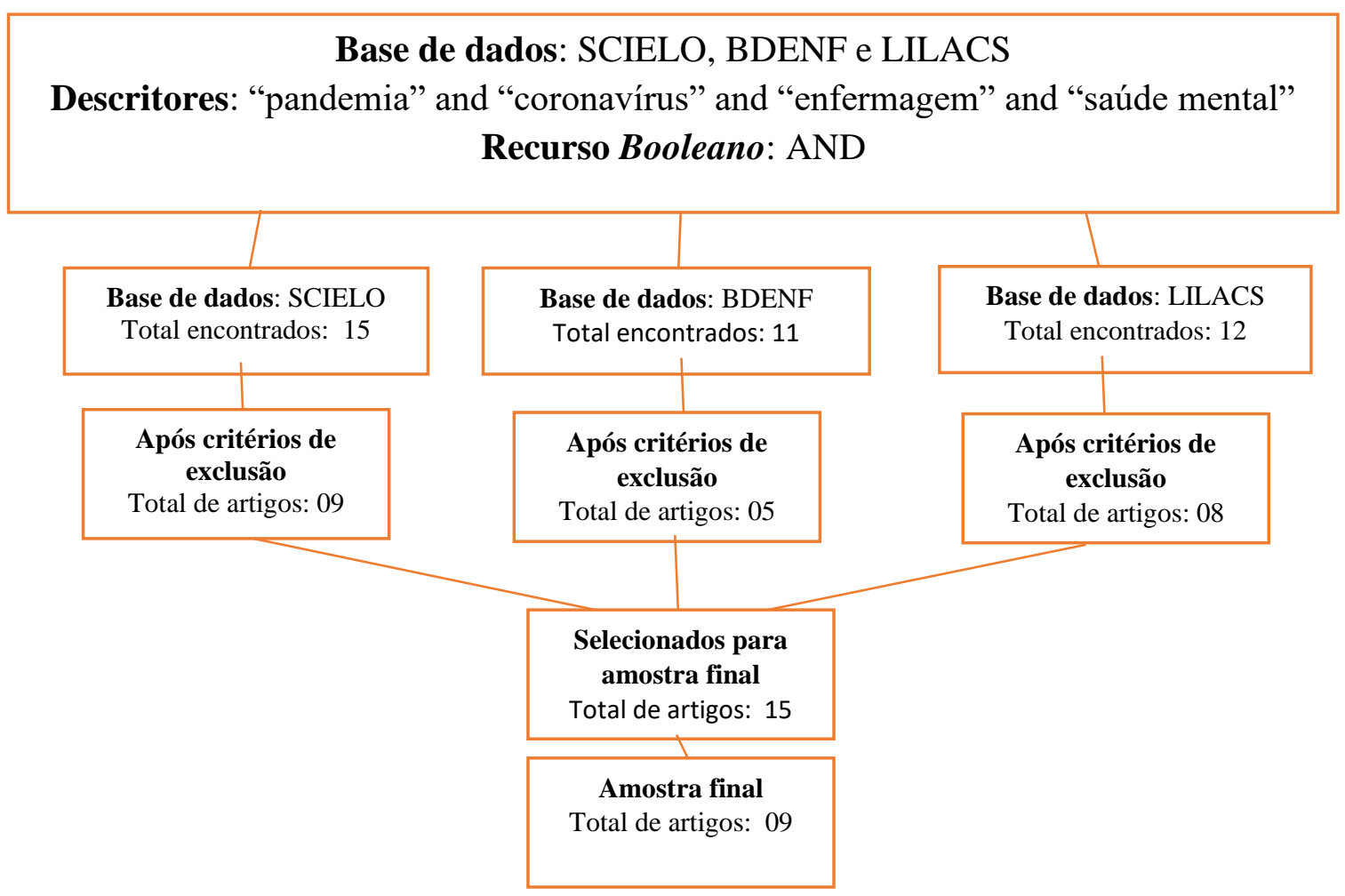

Fonte: Autores (2020).

\section{Resultados e Discussão}

Para composição deste estudo, foram pré-selecionados 15 estudos, sendo que destes 06 (seis) estudos foram excluídos após análise detalhada do escopo publicado (03 (três) estudos não apontam de fato os impactos na saúde física e mental para os enfermeiros frente sua atuação no combate a COVID-19 e os outros 03 (três) estudos não se enquadram no objetivo dessa pesquisa). Sendo assim a revisão se baseou em 09 (nove) artigos selecionados que atenderam aos parâmetros estabelecidos como critérios de inclusão referente aos impactos na saúde física e mental dos enfermeiros no enfrentamento da COVID-19. 04 (quatro) estudos pertencem à base de dados SCIELO, 02 (dois) a base BDENF e 03 (três) na base LILACS. O Quadro 1 apresenta a caracterização dos estudos.

De acordo com os dados coletados nos estudos que fazem parte desta revisão sistêmica integrativa, no que se refere a abordagem da temática 07 (sete) estudos discutem diretamente sobre os impactos da pandemia na saúde mental dos enfermeiros, 01 (um) estudo enfoca sobre a atuação dos profissionais no enfrentamento da COVID-19 e as repercussões na saúde, 01 (um) estudo evidencia sobre a importância de se pensar em estratégias para promoção da saúde mental dos enfermeiros diante dessa pandemia.

Em relação à base de dados que mais apresentou manuscritos destaca-se a base SCIELO (Scientific Electronic Library Online) tendo 04 artigos, publicados no intervalo de tempo entre os anos de 2020 e 2021, posteriormente a base de dados LILACS (Literatura Latino-Americana e do Caribe em Ciências da Saúde) com o total de 03 (três) artigos publicados em 2020, após segue a base de dados BDENF (Biblioteca Virtual de enfermagem) com apenas 02 (dois) artigos publicados no ano de 2020. Assim, observa-se que o ano de maior publicação foi o ano de 2020 com 77,77\% da amostra final, e lacunas de produção científica entre os anos de 2016 até 2019. 
Quadro 1 - Descrição dos artigos incluídos nesta revisão sistêmica integrativa. Maceió - AL, 2021.

\begin{tabular}{|c|c|c|c|c|}
\hline $\mathbf{N}^{\mathbf{o}}$ & Identificação título do artigo & $\begin{array}{c}\text { Tipo de } \\
\text { estudo/ano }\end{array}$ & Objetivo & Principais resultados \\
\hline 01 & $\begin{array}{l}\text { Trabalho de enfermagem na } \\
\text { pandemia da COVID-19 e } \\
\text { repercussões para a saúde mental } \\
\text { dos trabalhadores }\end{array}$ & $\begin{array}{l}\text { Estudo teórico- } \\
\text { reflexivo/2021 }\end{array}$ & $\begin{array}{l}\text { Refletir sobre o contexto de trabalho dos } \\
\text { profissionais de enfermagem na } \\
\text { pandemia da COVID-19 e as } \\
\text { repercussões para saúde mental desses } \\
\text { profissionais. }\end{array}$ & $\begin{array}{l}\text { Entre os principais fatores que eleva o impacto na saúde mental dos profissionais } \\
\text { da enfermagem são as condições precárias que envolvem a escassez qualitativa e } \\
\text { quantitativa de recursos materiais e humanos, enfatizando jornadas longas e } \\
\text { exaustivas; salários não condizentes; falta de EPIs; capacitação ineficaz; } \\
\text { fragilidades nos sistemas de saúde em tempos de crises endêmicas. }\end{array}$ \\
\hline 02 & $\begin{array}{l}\text { Saúde mental dos profissionais de } \\
\text { saúde no Brasil no contexto da } \\
\text { pandemia por COVID-19 }\end{array}$ & $\begin{array}{l}\text { Artigo } \\
\text { reflexivo/2021 }\end{array}$ & $\begin{array}{l}\text { Discutir as nuances que envolvem } \\
\text { desafios e Possibilidades da Saúde } \\
\text { Mental de profissionais de saúde no } \\
\text { Brasil, no contexto da atual pandemia } \\
\text { por COVID-19. }\end{array}$ & $\begin{array}{l}\text { Os impactos na saúde mental dos profissionais levam ao surgimento de doenças, } \\
\text { como a síndrome de Burnout, gerando assim respostas estressoras, exaustão } \\
\text { emocional e física, despersonalização entre outros. }\end{array}$ \\
\hline 03 & $\begin{array}{l}\text { Saúde mental dos profissionais de } \\
\text { enfermagem do Brasil no contexto } \\
\text { da pandemia COVID-19: ação do } \\
\text { Conselho federal de enfermagem }\end{array}$ & $\begin{array}{l}\text { Artigo } \\
\text { reflexivo/2020 }\end{array}$ & $\begin{array}{l}\text { Refletir sobre a saúde mental dos } \\
\text { profissionais de enfermagem brasileiros } \\
\text { no contexto da pandemia COVID-19 }\end{array}$ & $\begin{array}{l}\text { Através da realização do projeto de intervenção ao atendimento aos profissionais } \\
\text { da enfermagem que atuam na linha de frente a COVID-19, os sentimentos que } \\
\text { mais foram evidenciados pelos participantes foram: ansiedade, medo, } \\
\text { ambivalência, depressão e exaustão. }\end{array}$ \\
\hline 04 & $\begin{array}{l}\text { A saúde mental da enfermagem no } \\
\text { enfrentamento da COVID-19 em } \\
\text { um hospital universitário regional }\end{array}$ & $\begin{array}{l}\text { Estudo } \\
\text { observacional } \\
\text { transversal/2020 }\end{array}$ & $\begin{array}{l}\text { Identificar a prevalência e fatores } \\
\text { associados à ansiedade e depressão em } \\
\text { profissionais de enfermagem que atuam } \\
\text { no enfrentamento da COVID-19 em } \\
\text { hospital universitário. }\end{array}$ & $\begin{array}{l}\text { O estudo mostrou prevalência da ansiedade e depressão entre os profissionais de } \\
\text { enfermagem participantes, principalmente aqueles que trabalham na assistência, } \\
\text { dos } 88 \text { participantes obteve-se } 48,9 \% \text { com ansiedade e } 25 \% \text { depressão. }\end{array}$ \\
\hline 05 & $\begin{array}{l}\text { Projeto vida em quarentena: } \\
\text { estratégia para promoção da saúde } \\
\text { mental de enfermeiros diante da } \\
\text { COVID-19 }\end{array}$ & $\begin{array}{l}\text { Relato de } \\
\text { experiência/2020 }\end{array}$ & $\begin{array}{l}\text { Relatar a experiência } \\
\text { desenvolvimento do projeto de extensão } \\
\text { "Vida em Quarentena" com uma } \\
\text { estratégia para promoção da saúde } \\
\text { mental de enfermeiros atuantes na linha } \\
\text { de frente do combate à COVID-19 }\end{array}$ & $\begin{array}{l}\text { O artigo mostrou a necessidade de reconhecer a vulnerabilidade frente às } \\
\text { implicações mentais que os enfermeiros que atuam contra a COVID-19 estão } \\
\text { mais suscetíveis e a importância do planejamento de ações que proporcionem o } \\
\text { bem-estar físico e mental com a implementação de atividades que reforcem os } \\
\text { valores de crença, fé, apoio e esperança. }\end{array}$ \\
\hline 06 & $\begin{array}{l}\text { Repercussões da COVID-19 na } \\
\text { saúde mental dos trabalhadores de } \\
\text { enfermagem }\end{array}$ & $\begin{array}{l}\text { Teórico } \\
\text { reflexivo/ } 2020\end{array}$ & $\begin{array}{l}\text { Refletir acerca das repercussões da } \\
\text { COVID-19 na saúde mental dos } \\
\text { trabalhadores de enfermagem }\end{array}$ & $\begin{array}{l}\text { Enfatiza o alto risco de adoecimento mental dos profissionais da enfermagem em } \\
\text { tempos de pandemia, destacando com maiores repercussões o estresse } \\
\text { ocupacional, distúrbios psíquicos menores, síndrome de Burnout e o sofrimento } \\
\text { moral. }\end{array}$ \\
\hline 07 & $\begin{array}{l}\text { Atuação da enfermagem no cenário } \\
\text { da pandemia } \\
\text { COVID-19 }\end{array}$ & $\begin{array}{l}\text { Estudo descritivo, } \\
\text { exploratório do } \\
\text { tipo relato de }\end{array}$ & $\begin{array}{l}\text { Relatar as experiências, receios e anseios } \\
\text { dos profissionais de enfermagem que } \\
\text { atuam na linha de frente }\end{array}$ & $\begin{array}{l}\text { O estudo aponta que o enfrentamento ao cenário desconhecido proporcionou } \\
\text { preocupações, anseios e inseguranças aos profissionais tornando estes frágeis e } \\
\text { vulneráveis. }\end{array}$ \\
\hline
\end{tabular}


Research, Society and Development, v. 10, n. 8, e41410815695, 2021

(CC BY 4.0) | ISSN 2525-3409 | DOI: http://dx.doi.org/10.33448/rsd-v10i8.15695

\begin{tabular}{|c|c|c|c|c|}
\hline & & experiência/ 2020 & $\begin{array}{l}\text { aos cuidados de pacientes suspeitos e } \\
\text { confirmados da COVID- } 19 \text {. }\end{array}$ & \\
\hline 08 & $\begin{array}{l}\text { Saúde mental de profissionais de } \\
\text { enfermagem durante a } \\
\text { Pandemia de COVID-19: recursos } \\
\text { de apoio. }\end{array}$ & $\begin{array}{l}\text { Artigo } \\
\text { reflexivo/2020 }\end{array}$ & $\begin{array}{l}\text { Refletir sobre as implicações da } \\
\text { pandemia de coronavírus na saúde } \\
\text { mental dos profissionais de enfermagem } \\
\text { e os principais recursos de apoio em } \\
\text { desenvolvimento }\end{array}$ & $\begin{array}{l}\text { Entre os principais fatores de implicações na saúde mental dos profissionais de } \\
\text { enfermagem estão a alta demanda de atendimento; } \\
\text { exposição prolongada em ambientes críticos, risco contínuo de infecção, EPIs } \\
\text { desconfortáveis, limitadores e escassos. Entre as reações emocionais destacam } \\
\text { medo, alterações de humor, estresse, ansiedade. Entre as estratégias estão os } \\
\text { recursos de apoio, atendimento às necessidades básicas e autocuidado. }\end{array}$ \\
\hline 09 & $\begin{array}{l}\text { Condições de trabalho e o impacto } \\
\text { na saúde dos profissionais de } \\
\text { enfermagem frente a COVID-19 }\end{array}$ & $\begin{array}{l}\text { Artigo de } \\
\text { reflexão/2020 }\end{array}$ & $\begin{array}{l}\text { Refletir sobre as condições de trabalho } \\
\text { dos profissionais de enfermagem no } \\
\text { enfrentamento ao novo coronavírus e } \\
\text { apontar o impacto na vida desses } \\
\text { profissionais em meio à pandemia. }\end{array}$ & $\begin{array}{l}\text { Evidencia as más condições de trabalho, sobrecarga física e emocional, baixa } \\
\text { remuneração, ausência e desconfortos dos EPIs, além da necessidade de } \\
\text { valorização da classe. }\end{array}$ \\
\hline
\end{tabular}

Fonte: Dados da pesquisa (2021). 
Neste sentido, destaca-se entre as epidemias que já ocorrem entre os anos de 2016 até o presente momento, nenhuma provocou tanto impacto na saúde mental dos enfermeiros como a pandemia vivenciada atualmente, assim observa-se a necessidade de mais estudos científicos para área da saúde, pois constantemente ocorrem as modificações e a necessidade de atualização de práticas e medidas assistenciais condizentes com a realidade atual. Concomitantemente, às pesquisas científicas são importantes ferramentas frente a ampliação do conhecimento, acesso a informações produzido em nível nacional e internacional. Contribuindo assim, para uma atuação profissional e assistência de qualidade embasadas em evidências científicas (Reis et al., 2019).

Acerca do tipo de estudo observou-se a predominância do estudo reflexivo teórico compondo 06 estudos selecionados, 02 estudos são relato de experiência e apenas 01 estudo é observacional transversal. Grande parte dos estudos buscaram direcionar suas pesquisas objetivando refletir acerca das repercussões/impactos da COVID-19 na saúde mental dos enfermeiros, assim como relatar as experiências, detalhando os receios e anseios desses profissionais diante desse momento vivenciado e apenas 01 estudo foca em identificar os fatores associados ao surgimento da ansiedade e depressão nos enfermeiros em decorrência da pandemia. Ademais, no que se refere ao nível de evidência e do Qualis dos artigos utilizados para composição dessa revisão observa-se os seguintes dados evidenciados no Quadro 2 abaixo.

Quadro 2 - Apresentação dos estudos de acordo com o Nível de Evidência e Qualis. Brasil, 2021.

\begin{tabular}{|c|c|c|c|}
\hline $\mathbf{N}^{\mathbf{0}}$ & $\begin{array}{c}\text { BASE DE } \\
\text { INDEXAÇÃO }\end{array}$ & $\begin{array}{c}\text { NÍVEL DE } \\
\text { EVIDÊNCIA }\end{array}$ & QUALIS \\
\hline 01 & SCIELO & NÍVEL I & A2 \\
\hline 02 & SCIELO & NÍVEL I & B1 \\
\hline 03 & LILACS & NÍVEL I & B1 \\
\hline 04 & SCIELO & NÍVEL III & A2 \\
\hline 05 & BDENF & NÍVEL III & B2 \\
\hline 06 & BDENF & NÍVEL I & B2 \\
\hline 07 & LILACS & NÍVEL I & B2 \\
\hline 08 & SCIELO & NÍVEL III & B1 \\
\hline 09 & LILACS & NÍVEL I & B1 \\
\hline
\end{tabular}

Fonte: Dados da pesquisa (2021).

Quanto ao nível de evidência, pôde-se constatar que os estudos apresentam nível I correspondendo a 66,66\% das pesquisas analisadas, conforme apresentado no Quadro 2, e, com publicações realizadas em revistas de qualis B2 (33,33\%), B1 $(44,44 \%)$ e A2 (22,22\%). Face ao exposto, reitera-se que os estudos apresentam nível de evidência e qualis com o escopo de maior relevância no meio acadêmico.

\subsection{Repercussões da pandemia pela COVID-19 nos profissionais enfermeiros}

A partir dos estudos analisados e constituintes desta revisão, evidencia-se os impactos ocasionados pela pandemia da COVID-19 nos enfermeiros, desenvolvendo desta forma alterações psicológicas interferindo de forma notória no seu bem-estar físico e principalmente mental. Segundo Souza e Carvalho et al. (2021) e Ramos-Toescher et al. (2020) apontam que com a intensificação da rotina de trabalho gerando sobrecarga e desgastes físicos, além de muitas vezes dispor de baixa infraestrutura, a escassez de equipamentos de proteção individual - EPI’s, falta de treinamentos e capacitações acabam colocando o enfermeiro em uma situação maior de vulnerabilidade de contaminação e implicações na saúde física e psicológica. 
Outrossim, analisando o isolamento social como medida tomada no intuito de diminuir a propagação do vírus, Humerez et al. (2020) discute que direcionando a atenção às particularidades inerentes a cada realidade, enquanto a maioria da força de trabalho foram solicitados a trabalhar em casa, encontra-se os profissionais da saúde trabalhando em um cenário em que não se pode ter interação, comunicação e contato interpessoal, contribuindo assim para surgimento de diversos sentimentos negativos entre eles o tédio, solidão e tristeza, assim como a sensação de frustração e ineficiência.

Entre as implicações na esfera psicológica, Dantas (2021) evidencia que o receio de se contaminar, medo da perda dos familiares assim como de pacientes e colegas do trabalho, luto, isolamento social, pressão emocional são fatores que a cada dia elevam o número de profissionais diagnosticados com transtornos mentais, pois levam os mesmos, em especial os enfermeiros uma vez que estão mais expostos diretamente com pacientes infectados, ao confronto com seus próprios recursos psicológicos o que pode ser capaz de gerar um maior nível de estresse, ansiedade e depressão (Miranda, 2020).

Em uma pesquisa realizada em um hospital regional com os enfermeiros, o estudo evidenciou que a depressão é uma das principais implicações que acomete o bem-estar psicológico desses profissionais, ocorrendo com maior frequência no sexo feminino, o estudo ainda aponta que este quadro depressão é decorrente do cenário vivenciado e a necessidae de lidar constatimente com o sofrimento, a dor e a morte (Dal'bosco, 2020). Em contrapartida, Reis (2020) discute que muitas implicações psicológicas que afetam os profissionais da saúde, são negligenciadas e não dão as devidas atenção ou suporte emocionais necessários, provocando assim intensificação desses fatores e agravando ainda mais a saúde.

A síndrome de burnout abordada por Dantas (2021) representa uma implicação psicológica de caráter emergencial gerada por situações estressoras crônicas do setor de trabalho e exaustão extrema não resolvidas, que possui como principais características o cansaço físico e mental de forma excessiva, redução da produtividade no setor de trabalho, despersonalização, sentimentos negativos como a tristeza. Assim, em virtude da pandemia, reconhece-se os diferentes fatores que podem levar ao desenvolvimento da depressão, ansiedade assim como a síndrome de burnout do profissional enfermeiro, conforme corroboram Luz et al. (2020) ao referenciar sobre a necessidade de medidas que possam cuidar da saúde mental dos profissionais.

\section{Considerações Finais}

Diante desse contexto, compreende-se que os enfermeiros representam uma das principais classes que atuam na linha de frente contra a COVID-19 e que possuem grandes atribuições no combate e enfrentamento a esta pandemia, sabe-se ainda que muitas são as implicações e sentimentos gerados pela pandemia na população, entretanto na particularidade dos profissionais da saúde, em especial dos enfermeiros estas implicações também são experienciadas, todavia merecendo atenção e estratégias de combate às mesmas.

Ademais, ressalta-se que a saúde mental é um dos pilares importantes frente ao bom funcionamento do corpo e ao bem-estar de forma holística, logo as implicações provocadas pela pandemia da COVID-19 nessa esfera devem ser vistas e consequentemente criadas estratégias que possam contribuir com a promoção da saúde mental dos enfermeiros, desta forma planejando e implementando estratégias imediata possibilitando o rastreio das patologias que afetam as condições psicológicas, bem como que proporcionem o desenvolvimento de um ambiente de trabalho mais seguro e acolhedor, visando a redução dos impactos negativos gerados por este cenário pandêmico.

Logo este estudo possibilitou refletir sobre os impactos na saúde mental provocados pela pandemia da COVID-19 nos profissionais enfermeiros, trazendo à tona os fatores que levam ao adoecimento psicológico desses profissionais e como eles são experienciados, pois o mesmo evidenciou a fragilidade e a vulnerabilidade que os enfermeiros presenciam decorrente da natureza e as condições de trabalho que estão submetidos, consequentemente gerando diversos sentimentos estressores, alterações psicológicas, além de desgastes físicos e mentais. Assim, tendo em vista os achados, espera-se que novos estudos 
sobre a temática discutida sejam produzidos para maiores compreensões sobre o bem-estar físico e mental dos enfermeiros frente a pandemia da COVID-19 e assim possibilitando o desenvolvimento de estratégias que visem a promoção e o cuidado da saúde dessa classe.

\section{Referências}

Araujo, A. S. \& Comassetto, I. (2021). The nursing protagonism in the organization of health services during the COVID-19 pandemic. Research, Society and Development, 10 (1), e48110112014. https://rsdjournal.org/index.php/rsd/article/view/12014/10769

Araujo, A. S. et al. (2020). Interfaces on the suicide idea among universities in the health field: an integrative review. Braz. J. of Develop, 6 (3), 9610-9602, <https://www.brazilianjournals.com/index.php/BRJD/article/view/7252/6408>.

Chate, R. C. et al. (2020). Apresentação tomográfica da infecção pulmonar na COVID-19: experiência brasileira inicia. J. bras. Pneumol, 46 (2), 202-221, e2020012. https://doi.org/10.36416/1806-3756/e20200121

Cunha, M. C. (2015). Revisões de literatura: uma revisão com foco nas sistemáticas. CoDAS, 27(5), 409-410. https://doi.org/10.1590/2317-1782/20152275

Dal'bosco, E. B. et al. (2020). Mental health of nursing in coping with COVID-19 at a regional university hospital. Rev. Bras. Enferm, 73(2), e20200434. http://dx.doi.org/10.1590/0034-7167-2020-0434.

Dantas, E. S. O. (2021). Saúde mental dos profissionais de saúde no Brasil no contexto da pandemia por Covid-19. Interface - Comunicação, Saúde, Educação, 25, e200203. https://doi.org/10.1590/interface.200203.

Donato, H. \& Donato, M. (2019). Etapas na Condução de uma Revisão Sistemática. Acta Med Port, 32(3), 227-235. https://doi.org/10.20344/amp.11923

Humerez, D. C.et al. (2020). Saúde mental dos profissionais de enfermagem do Brasil no contexto da pandemia covid-19: ação do conselho federal de enfermagem. Cogitare Enfermagem, 25. http://dx.doi.org/10.5380/ce.v25i0.74115.

Luiz, E. M. F. et al. (2020). Repercussões da covid-19 na saúde mental dos trabalhadores de enfermagem. Revista de Enfermagem do Centro-Oeste Mineiro, 10 e3824. https://doi.org/10.19175/recom.v10i0.3824

Miranda, F. M. D’A. et al. (2020). Condições de trabalho e o impacto na saúde dos profissionais de enfermagem frente a covid-19. Cogitare Enfermagem, 25. http://dx.doi.org/10.5380/ce.v25i0.72702.

Oliveira, E. N. et al. (2020). Projeto Vida em Quarentena: estratégia para promoção da saúde mental de enfermeiros diante da COVID-19. Enfermagem em Foco,11(1). https://doi.org/10.21675/2357-707X.2020.v11.n1.ESP.3741.

Ramos-Toescher, A. M. et al. (2020). Saúde mental de profissionais de enfermagem durante a pandemia de COVID-19: recursos de apoio. Esc. Anna Nery, Rio de Janeiro, 24, e20200276. https://doi.org/10.1590/2177-9465-ean-2020-0276.

Reis, L. M. dos et al. (2020). Atuação da enfermagem no cenário da pandemia COVID-19. Revista nursing, 23(269), 4765-4768. https://doi.org/10.36489/nursing.2020v23i269p4765-4772

Saidel, M. G. B. et al (2020). Intervenções em saúde mental para profissionais de saúde frente a pandemia de Coronavírus. Revista Enfermagem UERJ, 28, e49923. https://doi.org/10.12957/reuerj.2020.49923.

Schmidt, B. et al. (2020). Saúde mental e intervenções psicológicas diante da pandemia do novo coronavírus (COVID-19). Estud. psicol. 37, e200063, https://doi.org/10.1590/1982-0275202037e200063.

Seixas, C. T. et al. (2021). A crise como potência: os cuidados de proximidade e a epidemia pela Covid-19. Interface (Botucatu), 25(1), e200379. http://dx.doi.org/10.1590/interface.200379.

Souza, N. V. D. O. (2021). Trabalho de enfermagem na pandemia da covid-19 e repercussões para a saúde mental dos trabalhadores. Rev. Gaúcha Enferm., Porto Alegre, 42, e20200225. https://doi.org/10.1590/1983-1447.2021.20200225.

Teixeira, C. F. de S. et al. (2020). A saúde dos profissionais de saúde no enfrentamento da pandemia de Covid-19. Ciênc. saúde coletiva, Rio de Janeiro, 25(9), 3465-3474. https://doi.org/10.1590/1413-81232020259.19562020.

Melo, G. A. A. et al. (2020). Benefícios da auriculoacupuntura em profissionais de enfermagem atuantes na COVID-19 à luz da Teoria do Conforto. Esc. Anna Nery, Rio de Janeiro, 24, e20200311. https://doi.org/10.1590/2177-9465-ean-2020-0311.

World Health Organization. (2020). Novel coronavirus (COVID-19). WHO. https:// https://www.who.int/emergencies/diseases/novel-coronavirus-2019

Whittemore, R. \& Knafl, K. (2005). The integrative review: updated methodology. JAdvNurs. 52(5), 546-53. 